Raida D. Mahmood

raida.1961@uomosul.edu.iq

Department of Mathematics,

College of Computer Science and

Mathematics

University of Mosul, IRAQ

Received on: 3/1/2012

\section{On SNF-rings , I}

\author{
Akram S.M. \\ Akr_tel@tu.edu.iq \\ Department of Mathematics, \\ College of Computer Science and Mathematics \\ University of Tikrit, IRAQ
}

Accepted on: 19/4/2012

\title{
ABSTRACT
}

A ring $R$ is called right SNF-rings if every simple right $\mathrm{R}$-module is $\mathrm{N}$-flat . In this paper , we give some conditions which are sufficient or equivalent for a right SNF-ring to be n-regular (reduced) .It is shown that

1- If $r(a)$ is a GW-ideal of $\mathrm{R}$ for every $a \in R$. then , $R$ is reduced if and only if $R$ is right SNFring.

2- If $R$ is an reversible, then $R$ is regular if and only if $R$ is right GQ-injective and SSNF-ring

Key words: SNF-rings, GW-ideal ,reversible.

\section{حول الحلقات من النمط I, SNF}

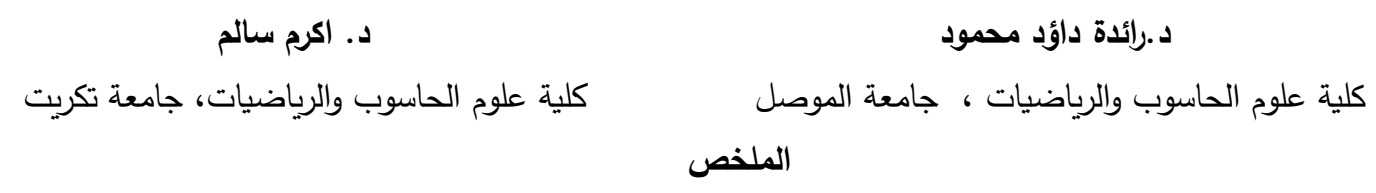

يقال للحلقة R حلقة يمنى من النمطSNF, إذا كان كل مقاس أيمن بسيط على الحلقة R مسطحاً من النمط N م ـ في

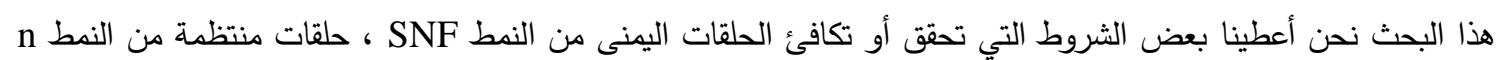

(مختزلة) • تبين لنا

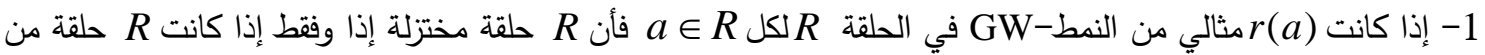

. SNF- النمط

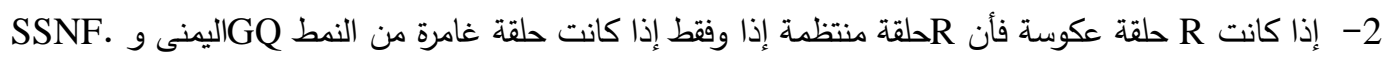

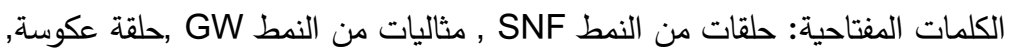

\section{Introduction}

Throughout this paper, $R$ denotes an associative ring with identity and all modules are unitary .We write $J=J(R)$ for the Jacobson radical of $R$, and $Y=Y(R)$ ( $Z=Z(R))$ for the right (left) singular ideal of $R$. The right and left annihilators of a subset $X$ of a ring $R$ are written as $r(X)$ and $l(X)$. A right $\mathrm{R}$-module $\mathrm{M}$ is said to be flat if, given any monomorphism $N \rightarrow Q$ of left R-modules $N$ and $Q$, the induced homomorphism $M \otimes N \rightarrow M \otimes Q$ is also monomorphism [1]. Generalizations on right flat modules have been studied by many authors (see [9] and[3]). In [5] SF rings are defined and studied. A ring $R$ is called right (left) SF-ring if every simple right (left) $\mathrm{R}$-module is flat . In [9], Wei and Chen first introduced and characterized a right N-flat modules, and gave many properties . A right $\mathrm{R}$-module is called $\mathrm{N}$-flat, if for any $a \in N(R)$, the map $I_{M} \otimes i: M \otimes R a \rightarrow M \otimes R$ is monic, where $i: R a \rightarrow R$ is the inclusion mapping. Actually, many authors investigated some properties of rings whose every simple right R-module is N-flat [4] and [9] .

Recall that a ring $R$ is called reduced ring if it has no non zero nilpotent elements, or equivalently, $a^{2}=0$, that implies $a=0$ for all $a \in R$. A ring $R$ is called reversible 
[2] if for $a, b \in R, a b=0$ implies $b a=0$. A ring $R$ is said to be Von Neumann regular (or just regular), if $a \in a R a$ for every $a \in R$ [5], a ring $R$ is called n-regular [6] if $a \in a R a$ for all $a \in N(R)$. Clearly, Von Neumann regular ring is n-regular, but the converse is not true by [6, Remark 2.19] .A ring $R$ is said to be right NPP if $a R$ is projective for all $a \in N(R)$ [6] . A right R-module $\mathrm{M}$ is called nil-injective if for any $a \in N(R)$, any R-homomorphism $R \rightarrow M$ can be extended to $R \rightarrow M$. Or equivalently there exists $m \in M$ such that $f(x)=m x$ for all $x \in a R$ [6,7]. Clearly, a reduced ring is right nil-injective, right NPP and n-regular ring [6] .

\section{SNF-ring}

Following [9], A ring $R$ is called right (left) SNF if every simple right (left) Rmodule is N-flat . proofs

The following lemma, which is duo to [9], plays a central role in several of our

\section{Lemma 2.1 :}

1- Let $B$ be a right R-module and there exists R-short exact sequence $0 \rightarrow K \stackrel{j}{\rightarrow} F \stackrel{g}{\rightarrow} B \rightarrow 0$ where $F$ is N-flat, then $B$ is N-flat if and only if $K \cap F a=K a$ for all $a \in N(R)$.

2- Let $I$ be a right ideal of $R$. then $R / I$ is $\mathrm{N}$-flat right R-module if and only if $I a=I \cap R a$ for all $a \in N(R)$.

3- $\quad$ Let $R$ be a ring then, $R$ is n-regular ring if and only if every right $\mathrm{R}$ - module is N-flat .

Following [5], a ring $R$ is called MERT ring if every maximal essential right ideal is a two-sided ideal of $\mathrm{R}$.

Clearly, a right SF-ring is right SNF-ring, but the converse is not true. Because there exists a reduced MERT ring which is not regular, there exists a reduced MERT ring $R$ which is not right SF by [12,Theorem 1]. On the other hand ,by [9, Theorem 4.7], reduced ring is right SNF,so there exists a right SNF-ring which is not right SF [9].

\section{Examples (3) :}

1- Let $Z_{2}$ be the ring of integer modulo 2 and let $G=\left\{g: g^{3}=1\right\}$ be acyclic group ,the group ring $Z_{2} G=\left\{0,1, g, g^{2}, 1+g, 1+g^{2}, g+g^{2}, 1+g+g^{2}\right\}$ is reduced nregular ring and SF-ring, therefore it is SNF-ring .

2- Let $Z_{2}$ be the ring of integer modulo 2 , then $R=\left\{\left[\begin{array}{cc}Z_{2} & Z_{2} \\ Z_{2} & Z_{2}\end{array}\right]\right\}$ isSNF-ring but not reduced .

3- The ring of integers $Z$ is SNF-ring but not SF-ring .

\section{Lemma 2.2: [2]}

Let $R$ be a reversible ring, then $r(a)=l(a)$ for all $a \in R$.

Following [7], a ring $R$ is said to be right (left) Nduo if $a R(R a)$ is an ideal of $R$ for all $a \in N(R)$.

\section{Proposition 2.3 :}


Let $R$ be a right $\mathrm{N}$ duo, SNF- ring, then $Y(R)=0$.

\section{Proof :}

Suppose that $Y(R) \neq 0$ then, $Y(R)$ contains a non-zero element $a$ such that $a^{2}=0$.let $x \in l(a), r \in R$. Since $R$ is right $\mathrm{N}$ duo, $a R$ is an ideal of $R$. Hence, $r a=a t$ for some $t \in R$. Therefore, $x r a=x a t=0$. This proves that $l(a)$ is a right ideal of $R$. Therefore, there exists a maximal right ideal of $R$ such that $l(a) \subseteq M$. Since $R$ is right SNF-ring and $a \in l(a) \subseteq M$, by lemma (2.1), there exists $b \in M$ such that $a=b a$, that is $(1-b) \in l(a) \subseteq M$ and so $1 \in M$, a contradiction. Therefore, $Y(R)=0$.

\section{Theorem 2.4:}

Let $R$ be a reversible ring.Then , $R$ is a right SNF-ring if and only if $R$ is nregular ring .

\section{Proof :}

Suppose that $R$ is n-regular, then $R$ is SNF-ring, lemma(2.1(3))

Conversely: Let $a \in N(R)$. We claim that $a R+r(a)=R$. If not, then there exists a maximal right ideal $M$ of $R$ such that $a R+r(a) \subseteq M$. Since $R$ is right SNF-ring, $R / M$ is an N-flat right R-module. By lemma (2.1), $a=x a$ for some $x \in M$. Since $R$ is reversible , $a=a x$. Hence , $(1-x) \in r(a) \subseteq M$ and so $1 \in M$, which is a contradiction. Therefore , $a R+r(a)=R$. Hence, $a b+z=1$ for some $b \in R$ and $z \in r(a)$. Since , $a z=0$ ,this gives $a=a b a$.Thus, $R$ is n-regular.

From Theorem (2.4) and definition of $C(R)$ we give the following Corollary :

\section{Corollary 2.5 :}

The center $(C(R))$ of any right (left) SNF-ring is n-regular ring.

Following [13], a left (right) ideal $L$ of a ring $R$ is called generalized weak ideal (GW-ideal), if for any $a \in L$, there existsn>0 such that $a^{n} R \subseteq L\left(R a^{n} \subseteq L\right)$.

\section{Theorem 2.6 :}

Let $R$ be a ring such that $r(a)$ is a GW-ideal of $R$ for every $a \in R$. Then , $R$ is reduced if and only if $R$ is right SNF-ring .

\section{Proof :}

Suppose $R$ is reduced, then $R$ is SNF-ring [9, Theorem 4.2] .

Conversely : Assume that $R$ is SNF-ring and $0 \neq b \in R$ such that $b^{2}=0$. Let $x \in l(b)$, then $b \in r(x)$. Since $\mathrm{r}(\mathrm{x})$ is a GW-ideal of $\mathrm{R}$ and $b^{2}=0$ we have $R b \subseteq r(x)$. This proves that $l(b)$ is a right ideal of $R$. Therefore, there exists a maximal right ideal $M$ of $R$ such that $l(b) \subseteq M$. Since $R$ is a right SNF-ring and $b \in l(b) \subseteq M$ by Lemma (2.1),$b=c b$ for some $c \in M, 1-c \in l(b) \subseteq M$ and so $1 \in M$, a contradiction , Therefore, $R$ is reduced.

Following [8] , a ring $R$ is called weakly normal if for all $a, r \in R$ and $e \in E(R)$, $e a=0$ implies areR is nil right ideal of $R$, where $E(R)$ stands for the set of all idempotent elements of $R$.

The following result is given in [8] 


\section{Lemma 2.7:}

Let $R$ be a weakly normal ring and $x \in R$.If $x$ is Von Neummanregular, then $x \in R x^{2} \cap x^{2} R$.

In the next result, we give another condition for SNF-ring to be a reduced ring .

\section{Theorem 2.8 :}

Let $R$ be a ring and every principal right ideal is a maximal .Then $R$ is reduced if and only if $R$ is right SNF-ring and weakly normal .

\section{Proof :}

Let $R$ be reduced, then it is clear $R$ is weakly normal , and SNF-ring .

Conversely : Let $a \in R$ with $a^{2}=0$ and every principal right ideal is a maximal, then $M=a R$. Since $R$ is right SNF-ring, $R / a R$ is an $\mathrm{N}$-flat right R-module .By Lemma (2.1) $a=b a$ for some $b \in a R$.Therefore, $a=a r a(b=a r)$ for some $r \in R$. By Lemma (2.7) , $a \in R a^{2}=0$, which implies $a=0$.Thus, $R$ is a reduced ring .

Next, we recall the following result of Wei and Chen [6] which proved the link between nil-injective and n-regular rings .

\section{Theorem 2.8 :}

The following conditions are equivalent for a ring $R$

$1-R$ is a n-regular ring .

2- Every left R-module is nil-injective .

3- Every cyclic left R-module is nil-injective .

4- $R$ is left nil-injective left NPP ring .

From Theorems (2.4 and 2.8) and Lemma (2.1), we get the following theorem .

\section{Theorem 2.19 :} injective

Let $R$ be a reversible ring. Then, $R$ is a right SNF-ring, if and only if $R$ is nil-

\section{3- Rings whose simple singular right $\mathbf{R}$-module are $\mathbf{N}$-flat}

In this section, we give an investigation of several properties for rings whose simple singular right $\mathrm{R}$-modules are $\mathrm{N}$-flat. Also, we study the relations between such rings and weakly regular ring .

\section{Definition 3.1 :}

A ring $R$ is said to be right SSNF-ring, if every simple singular right R-module is $\mathrm{N}$-flat .

\section{Theorem 3.2 :}

If $R$ is SSNF-ring with $l(a) \subseteq r(a)$, for every $a \in R$ then :

1- $Y(R) \cap Z(R)=0$

2- $Y(R) \cap J(R)=0$

\section{Proof :}

1) If $Y(R) \cap Z(R) \neq 0$, then there exists $0 \neq b \in Y(R) \cap Z(R)$ such that $b^{2}=0$. We claim that $R b R+r(b)=R$. Otherwise, there exists a maximal essential right ideal $M$ of $R$ containing $R b R+r(b)$. So, $R / M$ is a simple singular right R-module and 
then it is right $\mathrm{N}$-flat by hypothesis .Hence,$b=c b$ for some $c \in M$ (Lemma2.1), and so $(1-b) \in l(b) \subseteq r(b) \subset M$. Thus $1 \in M$, which is a contradiction . Therefore $1=x+y, x \in R b R, y \in r(b)$ and so $b=b x$. Since $R b R \subseteq Z(R), x \in Z(R)$. Thus $l(1-x)=0$ and $b=0$, which is a contradiction. Therefore $Y(R) \cap Z(R)=0$.

2) Suppose $Y(R) \cap J(R) \neq 0$, there exists $0 \neq b \in J(R) \cap Y(R)$ such that $b^{2}=0$, we will prove that $R b R+r(b)=R$. If not there exists a maximal right ideal $M$ of $R$ containing $R b R+r(b)$. Following the proof of (1) we get $b=b d$ for some $d \in R b R \subseteq J(R), b(1-d)=0$. Since $d \in J(R),(1-d)$ is invertible. This implies that $b=0$, which is a required contradiction. Therefore, $Y(R) \cap J(R)=0$.

Recall that a ring $R$ is right GQ-injective [11] if , for any right ideal $I$ isomorphic to a complement right ideal of $R$, every right R-homomorphism of $I$ into $R$ extends to an endomorphism of $R$. In [11], shows that if $R$ is right GQ-injective ring, then $J(R)=Y(R), R / J(R)$ is regular .

The next result is considered a necessary and sufficient condition for SSNF-rings to be regular ring .

\section{Theorem 3.3 :}

Let $R$ be reversible ring .Then, the following statements are equivalent :

1) $R$ is regular ring

2) $R$ is a right GQ-injective ring and right SSNF-ring .

\section{Proof :}

$1 \rightarrow 2$ Observe that if $R$ is regular then $R$ is n-regular and so every right R-module is $\mathrm{N}$-flat by [9, Theorem 4.2] .So we are done .

$2 \rightarrow 1$ From Theorem (3.2) $J(R) \cap Y(R)=0$. Since, $R$ is right GQ-injective, then $J(R)=Y(R)=0$ and $R$ is regular ring .

Following [7], a ring $R$ is called strongly min-able if every right minimal idempotent element is left semicentral .

\section{Theorem 3.4 :}

Let $R$ be a strongly right min-able, MERT ring. If $R$ is right SSNF-ring, then $R$ is a right weakly regular ring .

\section{Proof :}

We shall show that $R a R+r(a)=R$, for any $a \in N(R)$. Suppose that there exists $b \in N(R)$ such that $R b R+r(b) \neq R$. Then, there exists a maximal right ideal $M$ of $R$ containing $R b R+r(b)$. If $M$ is not essential in $R$. Then, $M$ is a direct summand of $R$ because $M$ is maximal. Now, we can write $M=r(e)$ for some $0 \neq e^{2}=e \in R$ and hence $e b=0$. Because $e R$ is a minimal right ideal of $R$ and $R$ is a strongly right minable ring,$b e=e b e=0$. Thus, $e \in r(b) \subseteq M=r(e)$, whence $e=0$. This is a contradiction. Therefore, $M$ must be an essential right ideal of $R$. Thus, $R / M$ is Nflat and so $b=c b$ for some $c \in M$ (Lemma 2.1), $1 \in M$ (R is MERT). a contradiction . Therefore, $R a R+r(a)=R$. In particular $x a y+z=1, x, y \in R, z \in r(a)$. So, axay $=a$. Hence, $R$ is a weakly regular ring .

\section{REFERENCES}


[1] Chose, S.U. (1960), Direct Products of Modules, Trans. Amer. Math. Soc. pp. 457-473.

[2] Cohn, P.M. ,(1999), "Reversible ring" ;Bull. London Math. Soc., 31, p.p 641-648.

[3] Mahmood, R.D. and Mohammed, H.Q.(2011), On N-flat rings, AL-Raf. J. of Computer Science and Math. Vol.8, No. 1, 71-77.

[4] Mahmood, R.D. and Younis, M.T.(2011), On n-regular rings, AL- Raf. J. of Computer science and Math. ,Vol.8 , No. 2 , 53-59 .

[5] Rege, M.B.(1986), On Von Neumann regular rings and SF-rings ,Math. Japonica , 31(6), 927-936 .

[6] Wei , J.C. and Chen, J.H. (2007), Nil-injective rings ,International Electronic Journal of Algebra, Vol.2:1-21 .

[7] Wei , J.C. and Libin , Li ,(2010), Nilpotent elements and reduced ring, Turk J. Math. , 34:1-13 .

[8] Wei , J.C. and Libin , Li ,(2011),Weakly normal rings , Turk J. Math. , $35: 1-11$.

[9] Wei , J. and Chen, J.(2008), NPP rings ,reduced rings and SNF rings, International Electronic Journal of Algebra, Vol. 4 , 9-26 .

[10] Wei , J. C. (2007), On simple singular YJ-injective modules , Sou. Asian Bull. of Math. ,31,1009-1018 .

[11] Yue Chi Ming, Roger , (1983), On quasi -injectivity and VonNeumann regularity, Moun. Math. 95, 25-32 .

[12] Zhang, J. and Du, X.N. (1993), Von Neumann regularity of SF - rings , Comm. Algebra, 21, 2445-2451.

[13] Zhou , H. (2007), Left SF-rings and regular rings , Comm. inAlgebra , $35,3842-3850$. 